\title{
Treatment of life-threatening wounds with a combination of allogenic platelet-rich plasma, fibrin glue and collagen matrix, and a literature review
}

\author{
MEHDI ASADI $^{1 *}$, DARYOUSH HAMIDI ALAMDARI ${ }^{2 *}$, HAMID REZA RAHIMI $^{3}$, MOHSEN ALIAKBARIAN $^{1}$, \\ ALI JANGJOO $^{1}$, ABBAS ABDOLLAHI ${ }^{1}$, MOSTAFA MEHRABI BAHAR ${ }^{1}$, ALI AZADMAND ${ }^{1}$, NASER FORGHANI ${ }^{1}$, \\ MOHAMMAD NORI SADEGH ${ }^{2}$, MOHAMMAD ESMAIL KHAYAMY ${ }^{4}$ and ALEXANDER SEIFALIAN ${ }^{5}$

\begin{abstract}
${ }^{1}$ Surgical Oncology Research Center, Imam Reza Hospital, Faculty of Medicine, Mashhad University of Medical Sciences;
${ }^{2}$ Stem Cell and Regenerative Medicine Research Group, Department of Biochemistry, Stem Cell Laboratory, Faculty of Medicine, Mashhad University of Medical Sciences; ${ }^{3}$ Student Research Committee, Department of Modern Sciences and Technologies, Faculty of Medicine, Mashhad University of Medical Sciences, Mashhad 917794-8564; ${ }^{4}$ Blood Transfusion Research Center, High Institute for Research and Education in Transfusion Medicine, Mashhad 91875, Iran; ${ }^{5}$ UCL Centre for Nanotechnology and Regenerative Medicine, Division of Surgery and Interventional Science, University College London, Royal Free Hampstead NHS Trust Hospital, London, UK
\end{abstract}

Received October 11, 2013; Accepted February 5, 2014

DOI: 10.3892/etm.2014.1747

\begin{abstract}
Currently there is no ideal procedure for the treatment of recalcitrant ulcers that are unresponsive to the majority of common treatments. However, several novel approaches have been proposed, including bone marrow stem cells, platelets, fibrin glue and collagen matrix. For the first approach treatment of a chronic wound, a non-invasive method is highly desirable. The present study was undertaken with the aim of evaluating the effect of a combination of platelets, fibrin glue and collagen matrix (PFC) in one treatment. A total of ten patients with aggressive, refractory, life-threatening wounds were recruited for the study and their treatment effects were evaluated. Initially, the ulcers were extensively debrided, measured and photographed at weekly intervals. The PFC combination was applied topically to the wound every two days. Following treatment, the wound was completely closed in nine patients and was markedly reduced in the other patient. The mean $100 \%$ healing time for the nine patients was $11.3 \pm 5.22$ weeks. There was no evidence of local or systemic
\end{abstract}

Correspondence to: Dr Daryoush Hamidi Alamdari, Stem Cell and Regenerative Medicine Research Group, Department of Biochemistry, Stem Cell Laboratory, Faculty of Medicine, Azadi Square, Pardis Campus, Mashhad University of Medical Sciences, Mashhad 917794-8564, Iran

E-mail:dhamidialam@yahoo.com

*Contributed equally

Key words: recalcitrant wounds, platelet-rich plasma, fibrin glue, collagen matrix complications or any abnormal tissue formation, keloid or hypertrophic scarring. Therefore, the results of the present study indicate that in the first approach, the combination of PFC components may be used safely in order to synergize the effect of chronic wound healing.

\section{Introduction}

Recalcitrant wounds are associated with increased morbidity and mortality, have a negative impact on the patient quality of life and pose a serious burden on the health care system $(1,2)$. The cost of treating non-healing wounds has been estimated to be $\$ 70,000$ (3). Considering other indirect costs, including absence from work, loss of employment, cost of medical transport, assistance with daily living and self-care and medication expenditures, it has been estimated that almost $\$ 5$ billion is spent annually in the USA on ulcer treatment (4). Therefore, promoting the acceleration of wound healing is highly desirable since patient quality of life is likely to improve and the economic impact on the health care system is likely to reduce.

The management of recalcitrant ulcers is a major challenge clinically. Current therapies include debridement, offloading and supplementary treatments. However, the response to treatment is often poor and the outcome disappointing. These wounds place a limb at risk of infection and amputation and also puts patients at risk of mortality. Considering that optimum wound healing requires the well orchestrated integration of complex biological and molecular events involved in cell migration, proliferation, extracellular matrix deposition and remodeling (5), several novel approaches have been proposed for recalcitrant ulceration treatment. These include the use of stem cells (6), platelet-derived growth factors (7) and fibrin glue (8). Each of these approaches has been reported to increase the response time of healing chronic wounds. 
In the present study, a combination of allogeneic platelets, fibrin glue and collagen matrix (PFC) was used for the treatment of life-threatening wounds.

In platelets, the bioactive factors are located in the $\alpha$-granules and dense granules. In tissue regeneration, the wound heals through three phases: Inflammation, proliferation and remodeling. The bioactive factors are active during each of these phases. The $\alpha$-granules contain growth factors and cytokines, including transforming growth factor- $\beta$, platelet-derived growth factor, insulin-like growth factor I and II, fibroblast growth factor, epidermal growth factor, vascular endothelial growth factor and endothelial cell growth factor. These growth factors and cytokines are important in cell proliferation, chemotaxis, cell differentiation and angiogenesis. The dense granules contain serotonin, histamine, dopamine, calcium and adenosine. These non-growth factors have fundamental effects on the biological aspects of tissue repair. Histamine and serotonin increase capillary permeability, which allows inflammatory cells greater access to the wound site and activates macrophages. Polymorphonuclear leukocytes migrate towards the area of inflammation, and soon thereafter, cells begin to proliferate while fibroblasts aid the formation of a ground substance. Adenosine receptor activation modulates inflammation during wound healing (10). Thus, the bioactive factors play a central role in the healing processes by modulating the recruitment, duplication, activation and differentiation of various cell types. Platelets are used in the form of platelet rich plasma (PRP) which is prepared in a two-step process. Whole blood is initially centrifuged to separate the plasma from the red blood cells and centrifuged again in order to separate the PRP from the platelet-poor plasma (11). Clinically valuable PRP contains at least one million platelets per $\mu 1$ (4-5 times more than the blood base line) (12). Lesser concentrations may not be relied on to enhance wound healing, and greater concentrations have not been shown to increase wound healing (13).

Fibrin glue is a topical biological adhesive, the effect of which mimics the final stages of coagulation, wherein thrombin splits off fibrinopeptide A and B from the fibrinogen chain to form a monomer, which polymerizes to form a fibrin clot at the site of application. Fibrin glue is a promising adjunct treatment in numerous surgical fields and is beneficial in procedures which involve a high risk of postoperative bleeding or the leakage of air, blood and other fluids (14). Fibrin clots provide an important temporary extracellular matrix for wound healing. Therefore, fibrin glue was used to apply an admixture of platelets to the wound. The structural composition of fibrin and the binding of fibrin to cells and proteins determines the wound healing process. This represents an ideal delivery vehicle for additional cells for the treatment of chronic wounds (8).

Collagen matrix functions as a scaffold for regeneration. When applied to a tissue defect, the sprouting of capillaries and the migration of fibroblasts into the collagen results in the induction of angiogenesis and fibroplasia. The efficacy of the collagen matrix has been demonstrated for the treatment of deep sacral ulcers (9).

In the present study, ten patients were recruited. The aim of the study was to evaluate the treatment of recalcitrant wounds using a combined application of PFC as a delivery vehicle for the sustained release of platelet-fibrinogen rich plasma (PFRP)-derived bioactive factors to stimulate healing in recalcitrant ulcers, where conventional treatment methods have failed. To the best of our knowledge, this is the first time that this combination has been applied for the treatment of chronic wounds.

\section{Patients and methods}

Patients. In total, ten patients with life-threatening recalcitrant ulcers, that did not respond to any conventional therapy, were included in this study. The patients exhibited various wound categories, including deep (undermined wounds, 4 cases; tunneling wounds, 5 cases) and superficial wounds (1 case). The study was conducted in accordance with the principles of the 1996 Declaration of Helsinki, with good standards of clinical practice. The study protocol, informed-consent forms and other study-related documents were reviewed and approved by the Human Research Ethics Committee of Mashhad University of Medical Sciences (Mashhad, Iran). All patients were able to read and understand, and willingly signed the informed-consent form for their participation in the study. Inclusion criteria included the presence of a recalcitrant wound (diabetic, vascular, compression or traumatic) for a minimum of three months. In addition, the patients had to be $>18$ years of age (both genders) and had to agree to comply with the protocol requirements, including the self-care of wounds and all follow-up visit requirements. Patients were excluded if they were pregnant, lactating mothers or were receiving or had received chemotherapy (within eight weeks of the study screening visit). In addition, patients were excluded if they were current participants in an additional clinical investigation or current candidates for vascular surgery, angioplasty or stenting. Patients presenting with the clinical characteristics of cellulitis at the ulcer site, purulence or sinus tracts that were unable to be removed by debridement of the wound, malignant wounds, vasculitis or connective tissue disease, bone marrow involvement (lymphoma-leukemia) and any systemic infection, or those being treated with corticosteroids were also excluded.

Platelet preparation. Platelets were prepared at the Blood Transfusion Organization (Mashhad, Iran). Great care was taken to exclude any contamination with the red blood cells, thus, only pure platelets that were well within their active period and were qualified, according to viral safety tests in accordance with blood transfusion regulations, were used.

The platelets and fibrin glue were prepared according to standard procedures. Following the collection of $400 \mathrm{ml}$ peripheral blood from the $\mathrm{ABO}$ match donor and transferring the sample to commercial 450-ml triple blood donation bags, the platelets were initially prepared by centrifugation at $2,000 \mathrm{x} \mathrm{g}$ for $2 \mathrm{~min}$ and a second centrifugation at 4,000 x g for $8 \mathrm{~min}$. Subsequently, the supernatant plasma was separated and $25 \mathrm{ml}$ PRP remained (15). The fibrinogen concentrate was prepared from separated plasma by two biochemical methods (16), the cryoprecipitation or the ethanol precipitation method. For the cryoprecipitation method, following a $-70^{\circ} \mathrm{C}$ freeze and a $4^{\circ} \mathrm{C}$ thaw, the plasma was centrifuged at $6,500 \mathrm{x} \mathrm{g}$ for $5 \mathrm{~min}$. The supernatant plasma was removed to leave a final volume of $25 \mathrm{ml}$. In the ethanol precipitation 


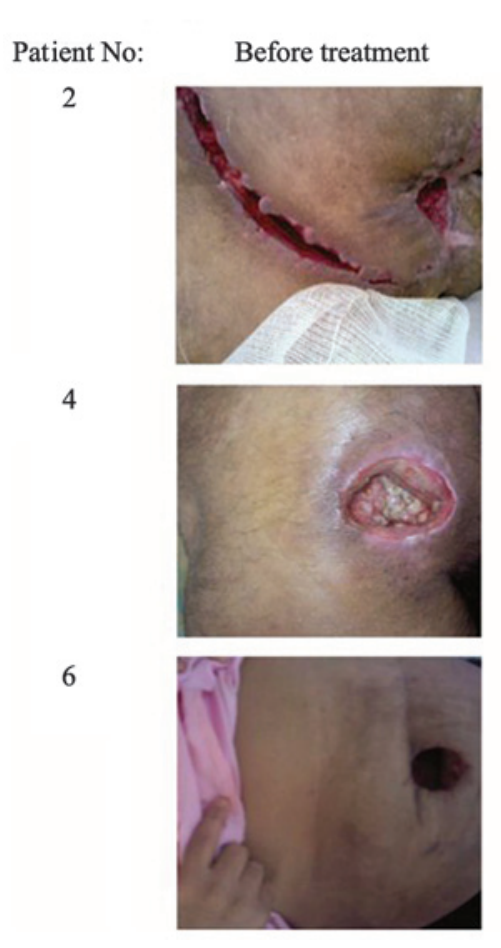

At the middle of treatment with allogenic plateletsrich plasma, fibrin glue and collagen matrix
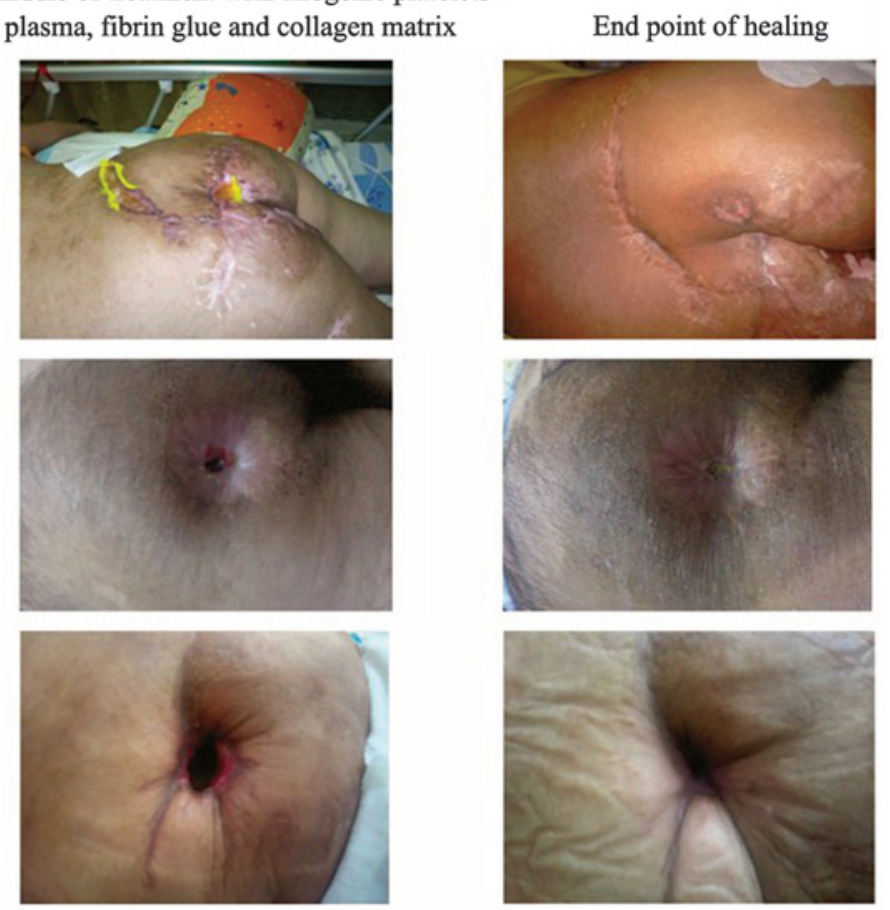

Figure 1. Images of wounds from certain patients prior to and following treatment with allogenic platelet-rich plasma, fibrin glue and collagen matrix.

method, absolute ethanol at $0^{\circ} \mathrm{C}$ was added to the plasma $(10 \% \mathrm{v} / \mathrm{v})$ and fibrinogen was obtained by centrifugation at $6,500 \mathrm{x} g$ for $15 \mathrm{~min}$. The supernatant plasma was removed to leave a final volume of $25 \mathrm{ml}$. Concentrated fibrinogen $(25 \mathrm{ml})$ was mixed with the platelets to form PFRP at a final volume of $50 \mathrm{ml}$. Thrombin $(1 \mathrm{ml})$ was prepared from the removed plasma by adding $10 \%$ calcium gluconate. Viral inactivation was performed for PFRP and thrombin by heating at $62^{\circ} \mathrm{C}$ for $1 \mathrm{~h}$. The samples were then frozen at $-20^{\circ} \mathrm{C}$ until required (for a maximum of three months). Prior to the application of PFRP, the necrotic and devitalized wound area was surgically debrided until the bleeding was recognized macroscopically. This allowed the PFRP to come into contact with viable wound tissue. When used, $50 \mathrm{ml}$ PFRP was mixed with $1 \mathrm{ml}$ thrombin and calcium gluconate. The collagen matrix (Surgicoll $^{\circledR}$; MBP, Medical Biomaterial Products, GmbH, Neustadt-Glewe, Germany) was immediately impregnated with this 51-ml PFRP solution and placed on the wound. Finally, paraffin gauze pads were placed over the wound and a bolster of rolled gauze pads were placed over the paraffin gauze. The dressing was subsequently wrapped with a rolled gauze. After two days, the entire dressing was removed and the wound was irrigated with isotonic sodium chloride solution. The wound was treated every two days, as aforementioned, for the formation of granulation tissue and closure. The patients were followed-up regularly for ulcer closure and any other possible complications. Images were captured on a digital camera on day 0 and at every three weeks, until the wound had healed. In certain cases, the ulcer volume was measured on day 0 , according to the volume of normal saline which filled the ulcer. In other cases, the ulcer dimensions (length $\mathrm{x}$ width $\mathrm{x}$ depth) were measured on day 0 . The effectiveness of PFRP-collagen application was evaluated after two months and in case of healing, the treatment continued until the wound closed.

\section{Results}

Overall analysis. Patient characteristics, medical history, wound size and the duration of the wound are presented in Table I. Following treatment, the wound was completely closed in nine patients and markedly reduced in the other patient. The mean $100 \%$ healing time for nine patients was $11.3 \pm 5.22$ weeks and the mean patient age was $45.1 \pm 1.03$ years. Overall, $70 \%$ of patients were male. There was no evidence of local or systemic complications or any abnormal tissue formation, keloid or hypertrophic scarring. Images of the wounds, prior to and following treatment, are presented in Fig. 1.

\section{Discussion}

In the present study, the effects of PFC combination treatment on ten patients with recalcitrant and life-threatening wounds were evaluated. The patients were critically ill with high fevers (even with the administration of antibiotics) and had not responded to traditional treatment modalities, including debridement, offloading and complementary therapies, such as antibiotic therapy, blood glucose level control, administration of zinc sulfate and multivitamins and the irrigation of the wound with normal saline and dressings. However, following treatment with the combination described, nine of the ten wounds were completely healed while the rest had improved markedly. In all the wounds, the ulcer bed rapidly demonstrated marginal in-growth, granulation tissue development, neovascularization and epithelialization. The appearance of ulcer bed bleeding during dressing changes 


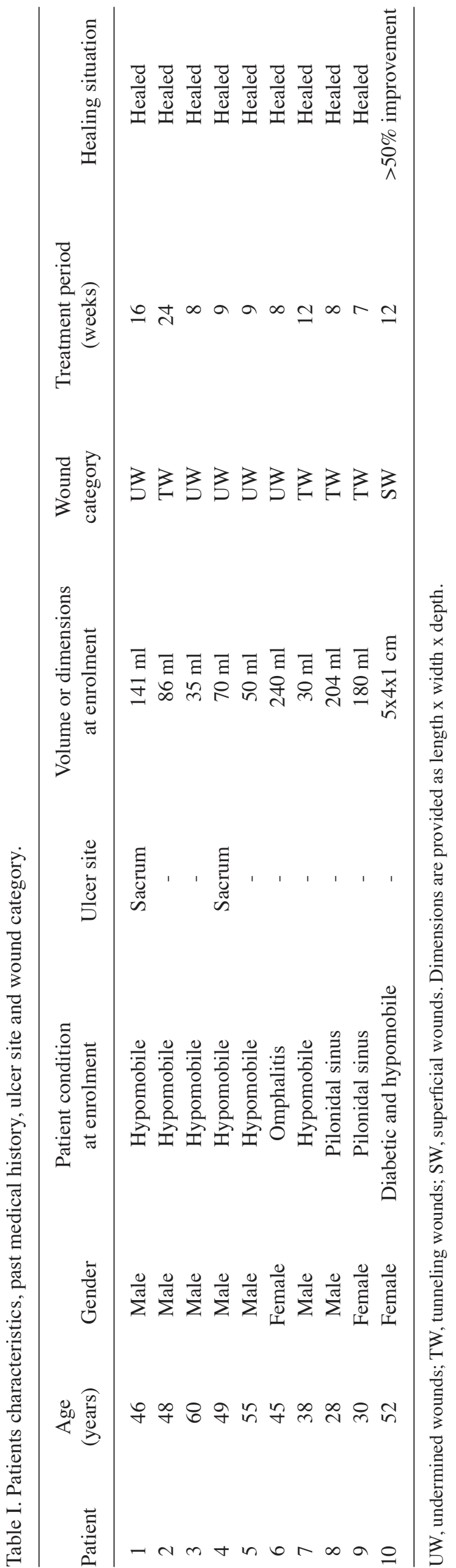

and ulcer palpation was observed and was deemed to be a positive indicator for wound healing.

In the present study, a control group was not selected since there was not a logical comparison to the patient group. The patients did not respond to any conventional or traditional therapies and the severe wound put the patient's life at risk, each patient was the self-control for themselves.

Although autologous and allogeneic PRP therapy is currently used clinically to stimulate tissue growth and regeneration and has been demonstrated to be effective in accelerating repair in chronic skin wounds $(17,18)$, the consensus on the therapeutic use of PRP remains controversial. In the literature, the majority of studies are performed on patients whose wounds have failed to heal with the use of conventional treatment techniques.

In the first clinical study, Knighton et al (1986) demonstrated that the topical application of growth factors promoted the healing of chronic cutaneous ulcers (7). Autologous platelet-derived wound healing factors (PDWHF) were used to treat 49 patients with chronic non-healing cutaneous ulcers. A multivariant analysis revealed a direct correlation between $100 \%$ healing with initial wound size and the initiation of PDWHF therapy (7).

In a prospectively randomized, blind trial by Knighton et al (19), 32 patients with chronic, non-healing, cutaneous wounds of the lower extremities were randomized and treated for eight weeks with autologous PDWHF or placebos, the end point of the study was the epithelialization of the wound. The results revealed that in the group who received treatment, $81 \%$ of patients exhibited epithelialization in eight weeks compared with $15 \%$ in the control group $(\mathrm{P}<0.0001)(19)$. In addition, the results demonstrated a highly statistically significant effect of topically applied PDWHF on the repair of chronic, non-healing, cutaneous ulcers (19).

In a pilot study by Atri et al (20), homologous platelet-derived wound healing factors (HPDWHFs) were used to treat recalcitrant ulcers in 23 patients with 27 persistent non-healing ulcers. The patients were initially subjected to controlled wound care for three months, with saline solution and silver sulfadiazine dressings. At the end of this period, persistent non-healing ulcers were treated by topical administration of HPDWHFs and silver sulfadiazine. Ulcer parameters were recorded on the first day and every week throughout the therapy until complete epithelization was achieved in either group. Each ulcer acted as its own control. In the controlled wound care group, only three ulcers in three patients achieved complete healing; the remaining 24 ulcers in 20 patients failed to achieve even $50 \%$ healing in the stipulated three-month period. However, when subjected to HPDWHF applications, these ulcers healed completely, with $100 \%$ healing occurring in $9.67 \pm 4.9$ weeks (range, 3-19 weeks), which was statistically significant $(\mathrm{P}<0.01)$. The healing response to HPDWHF applications was of uniform progression over the weeks. Only the basic cause of the ulcer determined the healing rates in this group. The shortest and the longest time to achieve $100 \%$ healing occurred in patients with diabetes $(6.88 \pm 2.97$ weeks) and in the venous stasis group (14.00 \pm 7.07 weeks) (20).

In a retrospective cohort study (with certain limitations) by Margolis et al (21), the effectiveness of autologous platelet releasate was assessed for the treatment of diabetic neuropathic 
foot ulcers in 26,599 patients. The results demonstrated that platelet releasate was more likely to be used in more severe wounds and was more effective in treating these wounds than the standard treatment.

In a prospective non-blinded study with 24 patients, Crovetti et al (2004) demonstrated the efficacy of once-weekly applications of autologous (three patients) or homologous origin (21 patients) platelet gel (PG) in healing cutaneous chronic wounds with various etiologies, including diabetes-related, vascular insufficiency, infectious disease, post-traumatic, neuropathic and vasculitis-related. At the time of publication, nine patients had healed completely, two patients had received cutaneous grafts, four patients had stopped treatment and nine patients had responded partially and were continuing to receive treatment (22). Although pain was reportedly reduced with the application of PG, neither patients nor clinicians were blinded to the treatment, possibly introducing bias to the self-report of pain (22).

A prospective, randomized, controlled multicenter trial in the USA by Driver et al (23), involving 40 patients with type 1 and type 2 diabetes (19 patients in the PRP group and 21 patients in the control group), reported the use of autologous PRP for the treatment of diabetic foot ulcers. The authors identified that $68.4 \%$ of patients in the PRP group (mean healing time, $42.9 \pm 18.3$ days) and $42.9 \%$ of patients in the control group (mean healing time, $47.4 \pm 22.0$ days) had wounds that healed (23).

A case study by McAleer et al (24), regarding a 57-year-old male with type 2 diabetes and a wound of six months duration, reported that the weekly use of autologous PRP was successful in healing a chronic lower extremity wound after four weeks.

In the case study by Knox et al (25), a 55-year-old male with a chronic non-healing decubitus ulcer of the sacrum, $>1$ year in duration, was treated with autologous PRP. The introduction of PRP therapy at week 14 led to a $26 \%$ reduction in wound depth over four weeks. At week 19, PRP therapy was combined with a powdered skin substitute to create a platelet-rich tissue graft. The combination led to marked results, eliminating wound tunneling and reducing the wound dimensions from $6.2 \times 6.7 \times 2.7-5.0 \times 6.0 \times 1.4 \mathrm{~cm}$ (length $\mathrm{x}$ width $\mathrm{x}$ depth) (25).

In the case report by Ficarelli et al (26), a chronic venous leg ulcer ( $8 \times 5 \mathrm{~cm}$ with raised margins) of a 79-year-old female almost completely healed following 20 allogenic PRP applications. Complete healing was observed one month following discontinuation with treatment.

A a pilot study by O'Connell et al (27), comprised a total of 21 patients, 12 patients with 17 venous lower-extremity ulcers and nine patients with 13 non-venous lower-extremity ulcers. The chronic lower-extremity ulcers were treated with autologous platelet-rich fibrin matrix membrane (PRFM). The primary endpoints were the incidence and time to complete closure, while the secondary endpoints were the incidence and time to $75 \%$ closure. Complete healing was achieved in $66.7 \%$ of the patients with venous lower-extremity ulcers in 7.1 weeks (median, six weeks) following an average of two applications of PRFM per patient. Of the non-venous lower extremity ulcer group, $44 \%$ of patients treated with PRFM healed completely during the study period (27).

In a perspective trial by Jeong et al (28) using 100 patients with diabetic foot ulcers, 52 patients were treated using a blood bank platelet concentrate and 48 patients (control group) were treated with topical fibrinogen and thrombin. Complete wound healing was achieved in $79 \%$ of the blood bank platelet concentrate-treated group and $46 \%$ of the control group $(\mathrm{P}<0.05)$. The times required for complete healing were $7.0 \pm 1.9$ and $9.2 \pm 2.2$ weeks in the blood bank platelet concentrate-treated and control groups, respectively $(\mathrm{P}<0.05)$. The degrees of wound shrinkage were $96.3 \pm 7.8$ and $81.6 \pm 19.7 \%$ for the treated and control groups, respectively $(\mathrm{P}<0.05)$. No adverse events associated with the study treatment were observed (28).

In a pilot study by Chen et al (29) involving 15 patients with 17 recalcitrant ulcers of various etiologies, the safety and efficacy of a combining allogeneic single-donor platelet and fibrin glue to enhance skin graft take for treating ulcers was evaluated. It was observed that the majority of the skin grafts were successful. The interval between the skin graft and complete wound healing ranged between 3 weeks and 2 months. No adverse reactions or recurrence of ulcers were observed during the three- to 18-month follow-up period (29).

In a pilot study by Marinacci et al, 7 patients were diagnosed with a diabetic ulcer with an extension of $>3.5 \mathrm{~cm}^{2}$. The ulcers were treated using autologous platelet and fibrin gel. Overall, four patients achieved total recovery of the ulcers, while three patients experienced a $>60 \%$ reduction in the ulcer diameter. The authors hypothesized that the recovery of the ulcers was associated with platelet activation in the specific ulcer area (21).

Sell et al (30) treated chronic stage IV pressure ulcers, in three veterans with spinal cord injury (SCI), with a sustained release PRP therapy to stimulate wound healing. PRP treatment consistently resulted in the formation of granulation tissue and improved the vascularity for each of the three patients, while reducing the overall ulcer area and volume. It was observed that the controlled release of growth factors from PRP has a positive stimulatory effect on the healing rate of chronic pressure ulcers in individuals with SCI (30).

A study by Greppi et al (31) on 11 hypomobile elderly patients with 14 chronic skin ulcers, who were unable to undergo autologous blood processing and had previously been ineffectively treated with expensive advanced medications for 8-275 weeks, evaluated the clinical efficacy of allogeneic PG prepared with standard blood banking procedures from routine platelet concentrates (PCs) obtained from buffy coats. No improvement was observed in three patients with 24,27 and $30 \mathrm{~cm}^{3}$ ulcers who were unable to be treated for any longer than 4,7 and 8 weeks due to progressively worsening clinical conditions. By contrast, 11 ulcers with a median size of $3.2 \mathrm{~cm}^{3}$ (range, 0.2-3.6 $\mathrm{cm}^{3}$ ) in the remaining 8 patients demonstrated $91 \pm 14 \%$ reduction following a median of 12 weeks (range, $1-20$ weeks). The cost of PG treatment (€19,976) amounted to $\sim 10 \%$ of the ineffective advanced medication hospital reimbursement fees $(€ 191,236)$. This study demonstrated the efficacy and feasibility of allogeneic PG for the treatment of recalcitrant ulcers in elderly hypomobile patients for whom autologous blood processing may be difficult (31).

Contrary to the aforementioned studies, in a randomized, prospective, double-blind, placebo-controlled study of topical autologous PDWHF in 18 patients [eight patients with nine wounds were treated with a placebo solution (controls) and 10 patients with 17 wounds were treated with PDWHF 
(treatment group)], Krupski et al (32) failed to demonstrate that autologous PDWHF provided additional benefit over traditional therapy for healing chronic non-healing cutaneous wounds.

It has been reported that collagen significantly promotes the repair process, particularly in the early stages. Collagen is used clinically with bone marrow stem cells by plastic surgeons in Japan for the treatment of chronic and acute wounds as a scaffold biomaterial (33-35).

In the pathophysiology of chronic non-healing wounds, the following causative factors have been documented: i) Significantly decreased local concentration, stability and bioavailability of bioactive factors; ii) significantly higher local activity of matrix metalloproteinases that degrade the extracellular matrix, impair tissue repair and suppress cell proliferation and angiogenesis $(35,36)$; and iii) phenotypically altered and/or senescent mesenchymal cells that fill the dermis of the skin (37). Accordingly, correction of the concentration of bioactive factors and wound matrix and reconditioning of phenotypically altered resident cells may lead to the success and efficacy of chronic wound healing.

In this study, rational beyond using PRP that it replenish the significantly decreased local concentration, stability and bioavailability of bioactive factors. PRP play a great role in host defense mechanism at the wound site by producing signaling proteins that attract macrophages (38), it also has anti-microbial activity against Escherichia coli and Staphylococcus aureus, including methicillin-resistant Staphylococcus aureus (39), Candida albicans (40) and Cryptococcus neoformans (40). . Fibrin glue, beyond behaving as a provisional matrix, actively recruits cells to trigger fibrin-mediated responses, including cell adhesion, migration, proliferation and tubule formation. Fibrin also promotes cell growth and vessel formation, which are beneficial during wound repair. Collagen has two roles, firstly functioning as a scaffold and secondly functioning as a sustained release method for the delivery of PRP growth factors and cytokines which are highly advantageous in the chronic wound state where senescent cells are prevalent (4) and may benefit from the sustained presence of stimulating factors.

In the present study, the patients required long-term treatment with platelets, for which large volumes of blood must be aspirated from patients. However, this was not feasible, thus, the allogenic platelets were replaced. All safety tests were performed for blood bank products, whilst paying attention to potential safety concerns. In addition to negative viral tests for the platelets which were used on the patients, viral deactivation was performed by heating.

According to the study by Ravari et al (41), it was hypothesized that for those patients who did not respond to the PFRP therapy, considering the pathological factors associated with the phenotypically altered and/or senescent mesenchymal cells in the wound, stem cell therapy should be considered as a second approach for the treatment of recalcitrant wounds. This hypothesis requires further study.

In conclusion, the results of the present study demonstrate the feasibility of the proposed practical model as a non-invasive method in the first approach for the treatment of life-threatening wounds. The use of PFRP-collagen therapy, involving a combination of sustained and immediate release of growth factors, healed or significantly reduced the wound size in the various categories of recalcitrant ulcer, including deep (undermined and tunneling wounds) and superficial wounds. There was no evidence of local or systemic complications associated with the procedure. Thus, on the basis of these results, the authors are currently performing a larger study with the aim of further evaluating PFRP-collagen therapy for the treatment of recalcitrant ulcers.

\section{Acknowledgements}

The study was supported by a grant from the Mashhad University of Medical Science Research Council (grant number for project number MUMS/88709). This paper is the thesis for Dr Ali Azadmand, the resident student of the General Surgery Department.

\section{References}

1. Brod M: Quality of life issues in patients with diabetes and lower extremity ulcers: patients and care givers. Qual Life Res 7: 365-372, 1998.

2. Singh N, Armstrong DG and Lipsky BA: Preventing foot ulcers in patients with diabetes. JAMA 293: 217-228, 2005.

3. Garber SL and Rintala DH: Pressure ulcers in veterans with spinal cord injury: a retrospective study. J Rehabil Res Dev 40: 433-441, 2003.

4. Medina A, Scott PG, Ghahary A and Tredget EE: Pathophysiology of chronic nonhealing wounds. J Burn Care Rehabil 26: 306-319, 2005.

5. Harding KG, Morris HL and Patel GK: Science, medicine and the future: healing chronic wounds. BMJ 324: 160-163, 2002

6. Badiavas EV and Falanga V: Treatment of chronic wounds with bone marrow-derived cells. Arch Dermatol 139: 510-516, 2003.

7. Knighton DR, Ciresi KF, Fiegel VD, Austin LL and Butler EL: Classification and treatment of chronic nonhealing wounds. Successful treatment with autologous platelet-derived wound healing factors (PDWHF). Ann Surg 204: 322-330, 1986.

8. Laurens N, Koolwijk P and de Maat MP: Fibrin structure and wound healing. J Thromb Haemost 4: 932-939, 2006.

9. Ichioka S, Kouraba S, Sekiya N, Ohura N and Nakatsuka T: Bone marrow-impregnated collagen matrix for wound healing: experimental evaluation in a microcirculatory model of angiogenesis, and clinical experience. Br J Plast Surg 58: 1124-1130, 2005.

10. Foster TE, Puskas BL, Mandelbaum BR, Gerhardt MB and Rodeo SA: Platelet-rich plasma: from basic science to clinical applications. Am J Sports Med 37: 2259-2272, 2009.

11. Gandhi A, Bibbo C, Pinzur M and Lin SS: The role of platelet-rich plasma in foot and ankle surgery. Foot Ankle Clin 10: 621-637, 2005.

12. Marx RE: Platelet-rich plasma (PRP): what is PRP and what is not PRP? Implant Dent 10: 225-228, 2001.

13. Mishra A, Woodall J Jr and Vieira A: Treatment of tendon and muscle using platelet-rich plasma. Clin Sports Med 28: 113-125, 2009.

14. Martinowitz U and Spotnitz W: Fibrin tissue adhesives. Thromb Haemost 78: 661-666, 1997.

15. Whitman DH, Berry RL and Green DM: Platelet gel: an autologous alternative to fibrin glue with applications in oral and maxillofacial surgery. J Oral Maxillofac Surg 55: 1294-1299, 1997.

16. Silver FH, Wang MC and Pins GD: Preparation and use of fibrin glue in surgery. Biomaterials 16: 891-903, 1995.

17. Alsousou J, Thompson M, Hulley P, Noble A and Willett K: The biology of platelet-rich plasma and its application in trauma and orthopaedic surgery: a review of the literature. J Bone Joint Surg Br 91: 987-996, 2009.

18. Anitua E, Sánchez M, Orive G and Andia I: Delivering growth factors for therapeutics. Trends Pharmacol Sci 29: 37-41, 2008.

19. Knighton DR, Ciresi K, Fiegel V, Schumerth S, Butler E and Cerra F: Stimulation of repair in chronic, nonhealing, cutaneous ulcers using platelet-derived wound healing formula. Surg Gynecol Obstet 170: 56-60, 1990. 
20. Atri SC, Misra J, Bisht D and Misra K: Use of homologous platelet factors in achieving total healing of recalcitrant skin ulcers. Surgery 108: 508-512, 1990.

21. Margolis DJ, Kantor J, Santanna J, Strom BL and Berlin JA Effectiveness of platelet releasate for the treatment of diabetic neuropathic foot ulcers. Diabetes Care 24: 483-488, 2001.

22. Crovetti G, Martinelli G, Issi M, et al: Platelet gel for healing cutaneous chronic wounds. Transfus Apher Sci 30: 145-151, 2004.

23. Driver VR, Hanft J, Fylling CP and Beriou JM: A prospective, randomized, controlled trial of autologous platelet-rich plasma gel for the treatment of diabetic foot ulcers. Ostomy Wound Manage 52: 68-70, 2006.

24. McAleer JP, Sharma S, Kaplan EM and Persich G: Use of autologous platelet concentrate in a nonhealing lower extremity wound. Adv Skin Wound Care 19: 354-363, 2006.

25. Knox RL, Hunt AR, Collins JC, DeSmet M and Barnes S: Platelet-rich plasma combined with skin substitute for chronic wound healing: A case report. J Extra Corpor Technol 38: 260-264, 2006

26. Ficarelli E, Bernuzzi G, Tognetti E, Bussolati O, Zucchi A, Adorni D and De Panfilis G: Treatment of chronic venous leg ulcers by platelet gel. Dermatol Ther 21: S13-S17, 2008.

27. O'Connell SM, Impeduglia T, Hessler K, Wang XJ, Carroll RJ and Dardik H: Autologous platelet-rich fibrin matrix as cell therapy in the healing of chronic lower-extremity ulcers. Wound Repair Regen 16: 749-756, 2008.

28. Jeong SH, Han SK and Kim WK: Treatment of diabetic foot ulcers using a blood bank platelet concentrate. Plast Reconstr Surg 125: 944-952, 2010.

29. Chen TM, Tsai JC and Burnouf T: A novel technique combining platelet gel, skin graft, and fibrin glue for healing recalcitrant lower extremity ulcers. Dermatol Surg 36: 453-460, 2010.

30. Sell SA, Ericksen JJ, Reis TW, Droste LR, Bhuiyan MBA and Gater DR: A case report on the use of sustained release platelet-rich plasma for the treatment of chronic pressure ulcers. J Spinal Cord Med 34: 122-127, 2011.

31. Greppi N, Mazzucco L, Galetti G, et al: Treatment of recalcitrant ulcers with allogeneic platelet gel from pooled platelets in aged hypomobile patients. Biologicals 39: 73-80, 2011.
32. Krupski WC, Reilly LM, Perez S, Moss KM Crombleholme PA and Rapp JH: A prospective randomized trial of autologous platelet-derived wound healing factors for treatment of chronic nonhealing wounds: a preliminary report. J Vasc Surg 14: 526-536, 1991.

33. Kouraba S, Sakamoto T, Yasuda M, Yamamoto Y and Kumakiri M Wound bed regeneration with bone marrow-impregnated collagen matrix for the treatment of the leg ulcer associated with cryoglobulinemia. J Jpn Plast Reconstr Surg 26: 825-830, 2006.

34. Mizuno H, Akaishi S, Koike S, Hyakusoku H and Miyamoto M: Clinical application of collagen matrix impregnated with bone marrow-derived mononuclear cells for ischemic refractory skin ulcers. J Jpn Plast Reconstr Surg 26: 726-732, 2006.

35. Vande Berg JS, Rudolph R, Hollan C and Haywood-Reid PL: Fibroblast senescence in pressure ulcers. Wound Repair Regen 6: 38-49, 1998.

36. Trengove NJ, Stacey MC, Macauley S, et al: Analysis of the acute and chronic wound environments: the role of proteases and their inhibitors. Wound Repair Regen 7: 442-452, 1999.

37. Mendez MV, Stanley A, Phillips T, Murphy M, Menzoian JO and Park HY: Fibroblasts cultured from distal lower extremities in patients with venous reflux display cellular characteristics of senescence. J Vasc Surg 28: 1040-1050, 1998

38. Lindeboom JAH, Mathura KR, Aartman IHA, Kroon FHM, Milstein DMJ and Ince C: Influence of the application of platelet-enriched plasma in oral mucosal wound healing. Clin Oral Implants Res 18: 133-139, 2007.

39. Bielecki T, Gazdzik T, Arendt J, Szczepanski T, Król W and Wielkoszynski T: Antibacterial effect of autologous platelet gel enriched with growth factors and other active substances: an in vitro study. J Bone Joint Surg Br 89: 417-420, 2007.

40. Tang YQ, Yeaman MR and Selsted ME: Antimicrobial peptides from human platelets. Infect Immun 70: 6524-6533, 2002

41. Ravari H, Hamidi-Almadari D, Salimifar M, Bonakdaran S, Parizadeh MR and Koliakos G: Treatment of non-healing wounds with autologous bone marrow cells, platelets, fibrin glue and collagen matrix. Cytotherapy 13: 705-711, 2011. 